\title{
Limb-bud and heart as a novel biomarker for gastric intestinal type adenocarcinoma
}

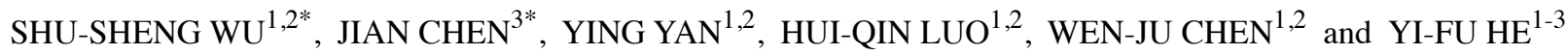 \\ ${ }^{1}$ Department of Oncology, West District of The First Affiliated Hospital of University of Science and Technology of China, \\ Division of Life Sciences and Medicine, University of Science and Technology of China; ${ }^{2}$ Department of Oncology, \\ Anhui Provincial Cancer Hospital, Hefei, Anhui 230031; ${ }^{3}$ Department of Oncology, The First Affiliated \\ Hospital of University of Science and Technology of China, Hefei, Anhui 230001, P.R. China
}

Received October 13, 2019; Accepted May 27, 2020

DOI: $10.3892 / \mathrm{ol} .2020 .11778$

\begin{abstract}
The present study compared the expression levels of limb-bud and heart (LBH) between gastric intestinal-type adenocarcinoma (GITA) and healthy gastric tissues; with the aim of investigating the possible effect of LBH on the prognosis of patients with GITA and to analyze the associated signaling pathways in GITA. Three Oncomine gastric datasets were utilized for the preliminary prediction of the expression levels of LBH mRNA in GITA and healthy gastric tissues. Gene expression and corresponding clinical data of 163 patients with GITA were downloaded from The Cancer Genome Atlas. Wilcoxon signed rank-sum test was used to distinguish the clinical value of $\mathrm{LBH}$ expression in the various clinicopathological features. Subsequently, Kaplan-Meier univariate and Cox multivariate survival analyses were performed to determine the prognostic significance of $\mathrm{LBH}$ expression in patients with GITA. Function enrichment analysis was conducted for the co-expression gene of $\mathrm{LBH}$, defined as correlation coefficient $r>0.06$ and $\mathrm{P}<0.05$ using Pearson's $\chi^{2}$ test. Bioinformatics data demonstrated that compared with that in the normal gastric mucosa, LBH mRNA expression was dramatically higher in GITA tissues $(\mathrm{P}<0.05)$.
\end{abstract}

Correspondence to: Dr Yi-Fu He, Department of Oncology, West District of The First Affiliated Hospital of University of Science and Technology of China, Division of Life Sciences and Medicine, University of Science and Technology of China, 107 Huanhudong Road, Hefei, Anhui 230031, P.R. China

E-mail: yifuhe@fsyy.ustc.edu.cn

*Contributed equally

Abbreviations: LBH, limb-bud and heart; GITA, gastric intestinal-type adenocarcinoma; TCGA, The Cancer Genome Atlas; GO, Gene Ontology; KEGG, Kyoto Encyclopedia of Genes and Genome

Key words: bioinformatics, limb-bud and heart, biomarker candidates, stomach neoplasms, The Cancer Genome Atlas
There were significant relationships between the differential expression levels of $\mathrm{LBH}$ and clinicopathological parameters in GITA patients (all $\mathrm{p}<0.05$ ), including pathological stage $\mathrm{T}$ (T3-4 vs. T1-2), lymph node metastasis (no vs. yes), distant metastasis (no vs. yes), histological grade (grade 3 vs. grades 1-2) and tumor stage (stages 3-4 vs. stages 1-2). Additionally, the overall survival and disease-free survival (DFS) of patients in the high expression group were poorer compared with those in the low expression group $(\mathrm{P}<0.05)$. Cox multivariate survival analysis indicated that increased LBH expression was an independent predictor of poor DFS prognosis in patients with GITA ( $\mathrm{P}=0.045)$. In summary, LBH is highly expressed in GITA, which can be used as an independent predictor of poor prognosis in patients with GITA. LBH co-expressed genes are closely associated with GITA tumor migration and metastasis.

\section{Introduction}

Globally, gastric cancer (GC) ranks as the fifth most diagnosed cancer the third leading cause of mortality associated with cancer (1). In 2018, 456,124 new cases of GC and 390,182 deaths were recorded in China, which accounted for $>50 \%$ of global GC deaths (2). Unfortunately, GC frequently remain undetected until advanced stages because initial symptoms are comparable to those of other diseases, such as chronic gastritis (3). Despite development of novel combined treatment strategies and increased understanding of the underlying mechanisms, the mortality rate of patients with GC remains relatively high (4). Therefore, there is an urgent need to investigate novel molecular pathways associated with the pathophysiology of GC so that innovative therapeutic intervention strategies for GC can be developed.

Lauren's classification, which divides GC into 'intestinal' and 'diffuse' types according to the morphological characteristics of the tumor, is frequently applied worldwide (5). Although reductions in the incidence of gastric intestinal-type adenocarcinoma (GITA) has been observed, it remains to be the most frequent type of $\mathrm{GC}$ found in high-incidence populations (6). The occurrence of GITA is a multi-step process that involves the transformation of the normal gastric mucosa to non-atrophic gastritis, multifocal atrophic gastritis, intestinal metaplasia, low-grade intraepithelial 
neoplasia, high-grade intraepithelial neoplasia and finally into carcinoma (7). Surmounting evidence has indicated that tumor cells reactivate underlying developmental processes to effectively perform this aforementioned multi-step process of tumorigenesis (8). However, the molecular mechanisms that regulate carcinogenesis and promote GITA tumorigenesis remain to be elucidated. A number of studies have previously indicated that transcription factors that are associated with embryonic development may serve vital roles in this pathological process (9-11).

Limb-bud and heart ( $\mathrm{LBH})$ is an important transcription cofactor involved in embryonic development and encodes a highly conserved nuclear protein that mediates transcriptional activation in mouse embryo tissue culture cells (12). Rieger et al (13) previously reported that $\mathrm{LBH}$ is a direct target of the Wnt signaling pathway during epithelial development, which was aberrantly overexpressed in highly invasive ER-negative, basal subtype human breast cancer types. In another study, Liu et al (14) demonstrated that LBH overexpression induced nasopharyngeal carcinoma cell cycle arrest during the G1/S transition and inhibited the growth of transplanted nasopharyngeal carcinoma tumors in vivo, by downregulating latent membrane protein-1-mediated NF- $\kappa \mathrm{B}$ transcriptional activity. Recently, Deng et al (15) reported that LBH expression was significantly downregulated in lung cancer tissue samples, where it associated with the prognosis and clinical characteristics of patients with lung cancer. Furthermore, knocking out the LBH gene has been found to promote the proliferation, migration and invasion of lung adenocarcinoma cells (15), where further bioinformatics analysis revealed that $\mathrm{LBH}$ was significantly associated with signaling pathways regulating cell adhesion (15). However, the role of LBH in GITA remain poorly understood.

Therefore, in the present study, The Cancer Genome Atlas (TCGA) and the Oncomine databases were used to compare the expression levels of LBH mRNA in GITA and healthy gastric tissues. In addition, the relationship between LBH gene expression and survival in patients with GITA was analyzed. Functional enrichment analysis was subsequently performed to examine the biological pathways associated with the $\mathrm{LBH}$ regulatory network in GITA.

\section{Materials and methods}

RNA sequencing of patient data and bioinformatics analysis. The Oncomine 4.5 database (https://www. oncomine. org/resource/login.html) is a publicly accessible online cancer microarray database and web-based data-mining platform containing 715 datasets and 86,733 samples. In the database, the gene was set as 'LBH', analysis type as 'cancer vs. normal analysis', cancer type as 'GITA' and the data type as 'mRNA'. Under this search conditions, three datasets including Chen Gastric, DErrico Gastric and Cho Gastric were used to predict the expression levels of LBH mRNA in GITA and healthy gastric tissues. Boxplots were then produced to compare the differences in the expression of LBH between GITA and healthy gastric mucosal tissues.

Expression profiles of GC samples and corresponding clinical information were downloaded from the official GDC portal of the TCGA database(https://portal.gdc.cancer. gov/, Data Release 16.0). Search keywords were as follows: Primary Site: stomach, Project: TCGA-STAD; Disease Type: adenocarcinomas, Data Category: transcriptome profiling, Experimental Strategy: RNA-Seq, Workflow Type: HTSeq-Counts. A total of 191 cases containing LBH gene expression information were downloaded (Tables SI and SII). Run ' $R$ ' software to normalize the data. At the same time, the clinical and pathological information including survival time was screened from the downloaded data, and diffuse gastric cancer cases were excluded. Combining data containing LBH gene expression information and clinicopathological characteristics data, a total of 163 GITA cases were obtained for further analysis. Boxplots were used to visualize expression differences of $\mathrm{LBH}$ according to discrete variables, including pathological stage $\mathrm{T}$, lymph node metastasis, distant metastasis, histological grade (16) and tumor stage (17).

Statistical analysis. All statistical analyses and figures were performed using R software (https://www.r-project.org/, version 3.5.0). The association between the baseline tumor characteristics of the patients in each group were assessed using Pearson's $\chi^{2}$ test. The association between pathological stage, lymph node metastasis, distant metastasis, histological grade and tumor stage in patients with GITA and LBH expression was analyzed using the Wilcoxon rank-sum test. Univariate survival analysis of patients with TCGA in relation to LBH expression were tested using the log-rank test. Kaplan-Meier survival analysis with log-rank test was used to compare the overall survival of the two groups. The Kaplan Meier-plotter database (18) was used to verify differences in the survival rates further. Cox regression was used for the multivariate analysis on the effects of LBH expression and clinical characteristics, including sex, age, histological grade and staging, on patient survival. $\mathrm{P}<0.05$ was considered to indicate a statistically significant difference.

Function enrichment analysis. Pearson's $\chi^{2}$ test was used to determine $\mathrm{LBH}$ co-expressed genes. The co-expressed gene of LBH was defined as the correlation coefficient $r>0.6$ and $\mathrm{P}<0.05$. Subsequently, in the $\mathrm{R}$ program, the 'cluster profiler' $(18,19)$ package was used to analyze and visualize gene ontology (GO) in the cellular component (CC), molecular function (MF) and biological process (BP) categories. Furthermore, Kyoto Encyclopedia of Genes and Genomes (KEGG, Release 90.0) pathway enrichment analysis was performed, where adjusted-P $<0.05$ was considered to indicate a statistically significant difference (17).

\section{Results}

LBH $m R N A$ overexpression in GITA. A total of 3 gastric datasets [Chen Gastric (20), DErrico Gastric (21) and Cho Gastric (22)] in the Oncomine database were used to examine the expression levels of LBH mRNA in GITA and healthy gastric mucosa tissues. In the datasets, the P-values and t-test results for the comparison of LBH mRNA levels in GITA and healthy gastric mucosa were as follows: $\mathrm{P}=1.21 \times 10^{-8}$, $\mathrm{t}$-test=6.216 (Chen Gastric dataset; Fig. 1A); $\mathrm{P}=4.14 \times 10^{-4}$, $\mathrm{t}$-test=3.625 (DErrico Gastric dataset; Fig. 1B); and $\mathrm{P}=0.030$, t-test=1.957 (Cho Gastric dataset; Fig. 1C). The expression 
A

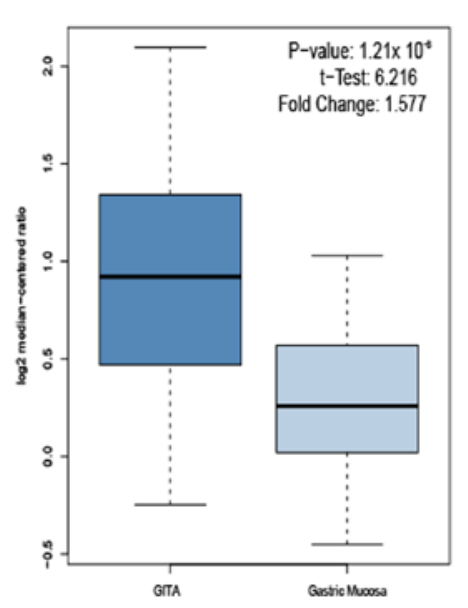

B

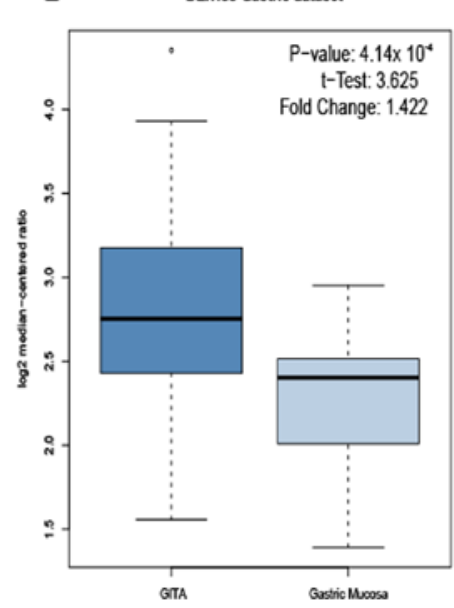

C

Cho Gastric dataset

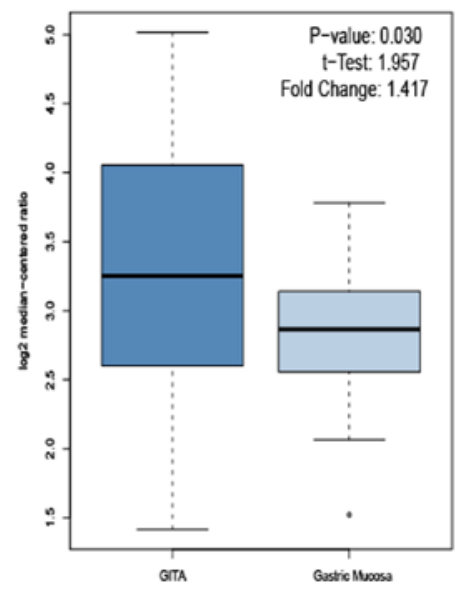

Figure 1. Comparison of limb-bud and heart expression between gastric intestinal type adenocarcinoma and gastric mucosa as predicted using the Oncomine database. Fold-change represents the fold difference in the expression of LBH mRNA in GITA and gastric mucosa. Results for the (A) Chen Gastric, (B) DErrico Gastric and (C) Cho Gastric datasets.

level of LBH mRNA in GITA tissue was significantly higher compared with that in healthy gastric tissue for all three datasets.

Association between LBH and clinicopathological parameters in patients with GITA. Among the 163 patients, 57 were male and 106 female. The age range was 30-90 years, in which 72 patients were aged $<67$ years and 88 patients were aged $>67$ years with three patients had unknown data for age. A total of 44 patients were in pathological stages T1-2 according to the American Joint Committee on Cancer Tumor-Node-Metastasis staging system (17), 117 in stages T3-4 and two exhibited unknown stages. Furthermore, 117 patients exhibited lymph node metastasis, 44 patients did not and 2 patients had unknown data for lymph node metastasis. A total of 14 patients exhibited distant metastasis, 147 patients did not and the data was unknown for 2 patients. Additionally, 90 patients were in histological grades 1-2 (17), 69 patients were in grade 3 and four patients had unknown grades. A total of 23 patients exhibited tumor stages I-II (17), 137 patients in stages III-IV whereas stages were unknown for 3 patients (Table I). Median overall survival (OS) was 17.18 months and median disease-free survival (DFS) was 8.71 months. Wilcoxon rank-sum test was used to distinguish the LBH expression in patients stratified into the different groups according to the clinicopathological parameters (Fig. 2). The expression of LBH was found to be increased in patients in pathological stage T3-4 (T3-4 vs. T1-2; $\mathrm{P}=0.004$ ), no lymph node metastasis (no vs. yes; $\mathrm{P}=0.003$ ), no distant metastasis (no vs. yes; $\mathrm{P}=0.024$ ), histological grade 3 (grade 3 vs. grades $1-2 ; \mathrm{P}=0.025$ ) and tumor stage III-IV (III-IV vs. I-II; P<0.001). These results suggest that the elevated expression of LBH is associated with advanced tumor staging and poor differentiation.

Increased LBH expression is associated with poor survival in GITA. The prognostic significance of LBH expression levels in patients with GITA was next investigated. TCGA data of 163 patients was divided into high-expression $(n=83)$ and the low-expression $(n=80)$ groups using the median expression of $\mathrm{LBH}$ value as the cut-off value.

Kaplan-Meier survival analysis with log-rank test was used to compare the overall survival of the two groups. The hazard ratios (HRs) of the two groups were calculated using univariate Cox regression analysis. The results demonstrated that patients in the low expression group had significantly prolonged OS and DFS compared with those in the high expression group (both $\mathrm{P}<0.05$; Fig. $3 \mathrm{~A}$ and $\mathrm{B}$ ). The $\mathrm{HR}$ in the high and low LBH expression groups were 1.454 [95\% confidence interval $(\mathrm{CI})=1.077-1.963]$ and $1.689(95 \% \mathrm{CI}=1.134-2.516)$, respectively, as per the univariate Cox regression analysis. Additionally, using the cut-off value established as aforementioned, further verification in the Kaplan Meier-plotter database demonstrated that patients in the low expression group exhibited prolonged OS and DFS compared with those in the high expression group (both, $\mathrm{P}<0.05$; Fig. $3 \mathrm{C}$ and $\mathrm{D}$ ). The $\mathrm{HR}$ in the high and low-expression groups were 1.25 (95\% $\mathrm{CI}=1.06-1.48)$ and 1.28 (95\% CI=1.04-1.56), respectively, according to the univariate Cox regression analysis.

To evaluate the independent prognostic value of $\mathrm{LBH}$ expression, multivariate Cox regression analysis was performed. The results indicated that $\mathrm{LBH}$ expression was independently associated with DFS $(\mathrm{P}=0.045 ; \mathrm{HR}=1.53$; 95\% CI=1.01-2.3; Fig. 4). However, LBH was not found to be independently associated with $\mathrm{OS}(\mathrm{P}=0.078 ; \mathrm{HR}=1.34$; 95\% CI=0.97-1.8; Fig. 5), but tumor stage $(\mathrm{P}=0.047 ; \mathrm{HR}=4.31$; $95 \% \mathrm{CI}=1.02-18.2)$ and age $(\mathrm{P}=0.026 ; \mathrm{HR}=1.84 ; 95 \%$ $\mathrm{CI}=1.07-3.1)$ were independent prognostic indicators of OS (Fig. 5).

Functional enrichment analysis of the LBH co-expression network. A total of 258 genes, which differentially expressed as a result of CENPK alteration, were screened using the threshold of absolute Pearson's $r>0.6$ (Table SIII). The GO enrichment analysis of the co-expressed mRNA indicated that 'collagen-containing extracellular matrix (ECM)', 'ECM structural constituent' and 'ECM organization' were the most significant categories of enriched $\mathrm{CC}, \mathrm{MF}$ and $\mathrm{BP}$ 
Table I. Baseline patient and tumor characteristics in each group.

\begin{tabular}{|c|c|c|c|c|}
\hline \multirow[b]{2}{*}{ Characteristic } & \multirow[b]{2}{*}{ No. of patients, n (\%) } & \multicolumn{2}{|c|}{ LBH expression, $\mathrm{n}(\%)$} & \multirow[b]{2}{*}{ P-value } \\
\hline & & High & Low & \\
\hline \multicolumn{5}{|l|}{ Age, years } \\
\hline$<67$ & $72(44.2)$ & $37(51.4)$ & $35(48.6)$ & 0.975 \\
\hline$>67$ & $88(54.0)$ & $45(51.1)$ & 43 (48.9) & \\
\hline \multicolumn{5}{|l|}{ Sex } \\
\hline Male & $57(35.0)$ & $33(58.0)$ & $24(42.0)$ & 0.192 \\
\hline Female & $106(65.0)$ & $50(47.2)$ & $56(52.8)$ & \\
\hline \multicolumn{5}{|c|}{ Histological grade } \\
\hline Grade $1-2$ & $90(55.2)$ & $43(47.8)$ & $47(52.2)$ & 0.465 \\
\hline Grade 3 & $69(42.3)$ & $37(53.6)$ & $32(46.4)$ & \\
\hline \multicolumn{5}{|l|}{ Tumor stage } \\
\hline Stage I-II & $23(14.1)$ & $11(47.8)$ & $12(52.2)$ & 0.772 \\
\hline Stage III-IV & $137(84.0)$ & $70(51.1)$ & $67(48.9)$ & \\
\hline \multicolumn{5}{|c|}{ Pathological stage $\mathrm{T}$} \\
\hline $\mathrm{T} 1-2$ & $44(27.0)$ & $20(45.5)$ & $24(54.5)$ & 0.394 \\
\hline $\mathrm{T} 3-4$ & $117(71.8)$ & $62(53.0)$ & $55(47.0)$ & \\
\hline \multicolumn{5}{|c|}{ Lymph node metastasis } \\
\hline Yes & $117(71.8)$ & $61(52.1)$ & $56(47.9)$ & 0.809 \\
\hline No & $44(27.0)$ & $22(50.0)$ & $22(50.0)$ & \\
\hline \multicolumn{5}{|c|}{ Distant metastasis } \\
\hline Yes & $14(8.6)$ & $7(50.0)$ & $7(50.0)$ & 0.981 \\
\hline No & $147(90.2)$ & $74(50.3)$ & $73(49.7)$ & \\
\hline
\end{tabular}

LBH, limb-bud and heart; T, tumor depth.
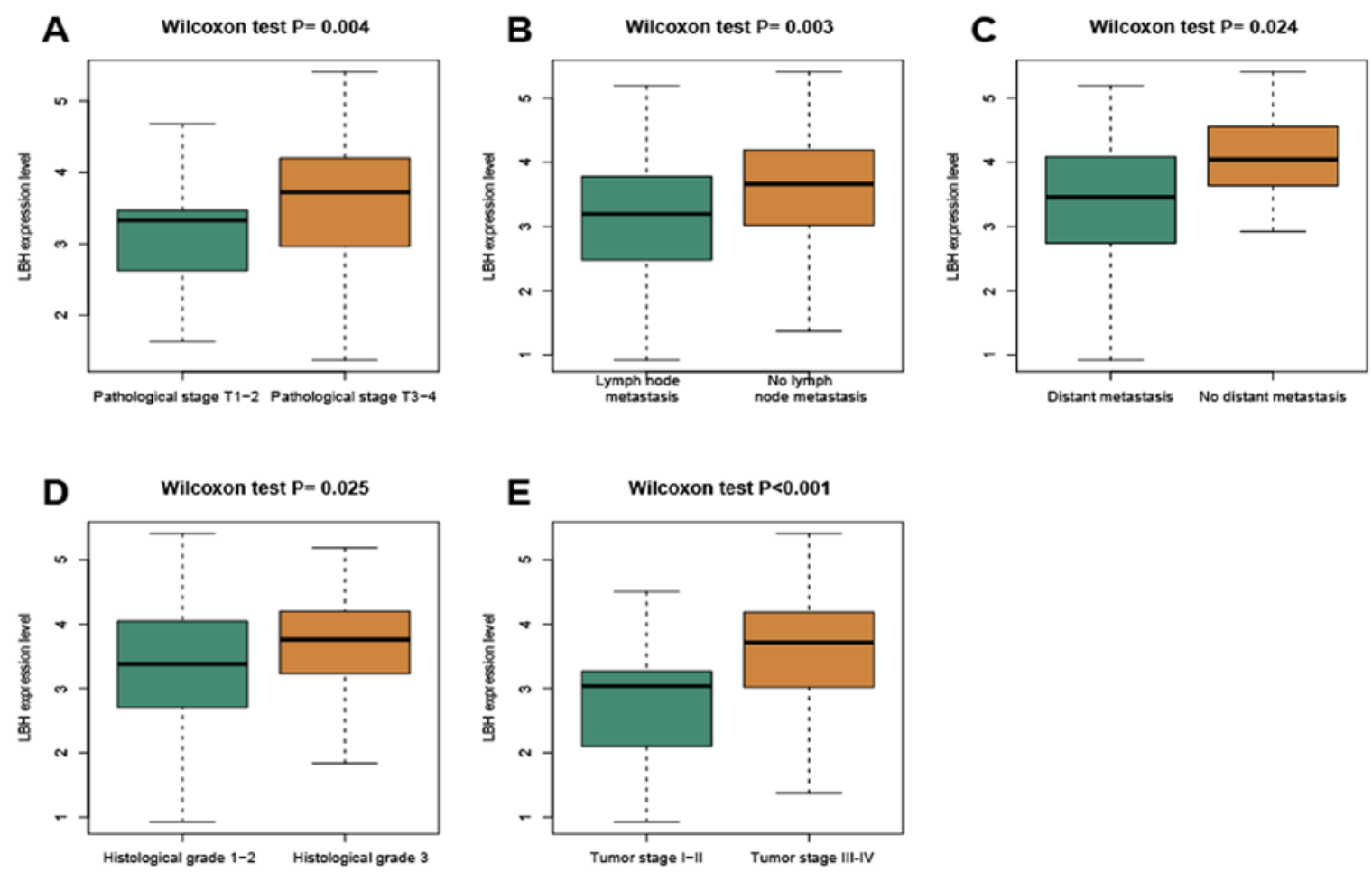

Figure 2. Association between LBH expression and clinicopathological characteristics, generated using R software. Comparison of LBH expression in samples of patients in different categories of (A) pathological stage T, (B) lymph node metastasis, (C) distant metastasis, (D) histological grade and (E) tumor stage. LBH, limb-bud and heart; T, tumor depth. 
A

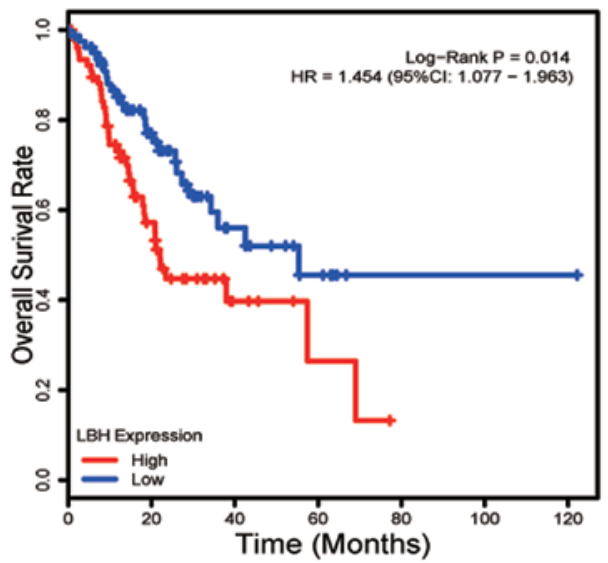

C

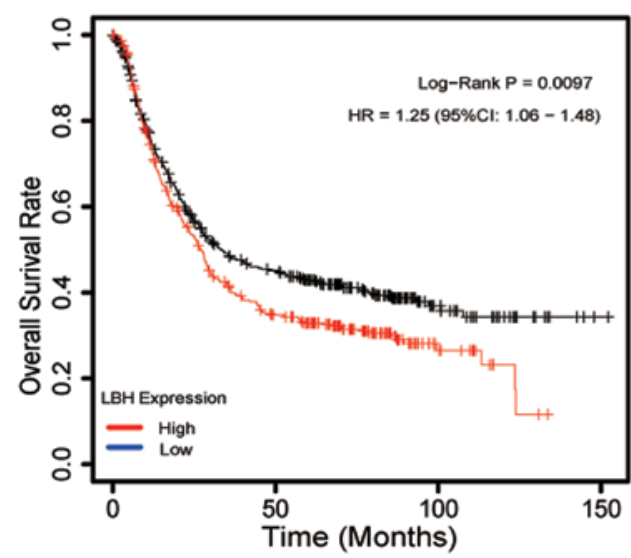

B

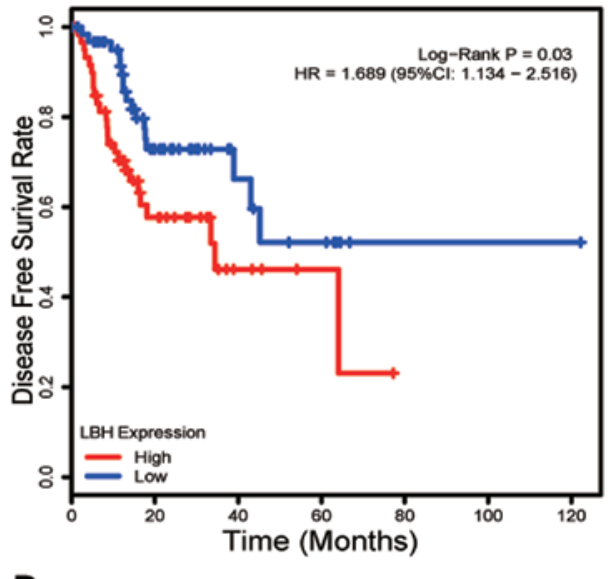

D

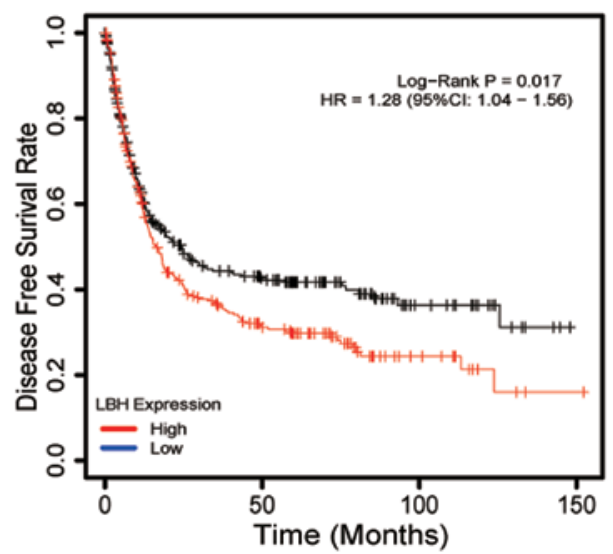

Figure 3. High expression of LBH is associated with worse prognosis in patients with gastric intestinal type adenocarcinoma. Data from The Cancer Genome Atlas database indicated that the (A) overall survival and (B) disease free survival rates were lower in patients in the high LBH expression group compared with those in the low expression group. Additionally, data from Kaplan-Meier plotter database demonstrated that (C) overall survival and (D) disease free survival rates were lower in patients in the high LBH expression group compared with those in the low expression group. LBH, limb-bud and heart; HR, hazard ratio; CI, confidence interval.

(Fig. 6A-C; Table SIII). The KEGG pathway enrichment analysis indicated that 'focal adhesion' and 'ECM-receptor interaction' were the most significant enrichment pathways (Fig. 6D; Table SIV). Additionally, LBH is involved in a series of cancer-related biological processes or signaling pathways, including proteoglycans in cancer and phosphatidylinositol 3-kinase/protein kinase B (PI3K-AKT) signaling pathways in tumors.

\section{Discussion}

LBH is a highly conserved and tissue-specific transcription regulator that serves an essential role in the embryonic development of vertebrates (12). Embryonic development and tumorigenesis have been reported to exhibit similar molecular mechanisms (23). LBH is a direct target of the $\mathrm{Wnt} / \beta$-catenin signaling pathway (13), which is fundamental to the genetic network for stem cell control and oncogenesis in various epithelial tissues, such as colorectal and breast tissue (24). Ashad-Bishop et al (25) previously revealed that LBH is required for Wnt-induced mammary hyperplasia and tumor formation. Reduced mammary hyperplasia in LBH-deficient mouse mammary tumor virus-Wnt1 mice at pre-neoplastic stages was associated with reduced cell proliferation and increased cell death, suggesting that LBH promoted mammary epithelial cell hyperproliferation. Lindley et al (26) demonstrated that $\mathrm{LBH}$ is an essential regulatory factor for the expansion and maintenance of basal multifunctional breast stem cells (MaSC), acting upstream of the $\Delta \mathrm{Np} 63$ oncogene to promote a multipotent basal MaSC state and repress luminal differentiation. In another previous study, Chen et al (27) demonstrated that high levels of LBH expression could be detected in 20/226 (8.8\%) of hepatocellular carcinoma (HCC) samples. The mean survival time was prolonged in patients with HCC who exhibited low LBH expression compared with those with high expression. Therefore, LBH overexpression may contribute to the development and progression of HCC.

These previous studies aforementioned have implied that $\mathrm{LBH}$ may function as an oncogene, which appeared to be consistent with the findings of the present study. Elevated expression of LBH in GITA tissues compared with that in normal gastric mucosa was found using Oncomine and TCGA public databases. The differential expression levels of $\mathrm{LBH}$ were found in patients with GITA who were stratified according to the clinicopathological parameters, including pathological stage T, lymph node metastasis, distant metastasis, histological grade and tumor stage. Subsequently, the association between LBH expression and prognosis in patients with GITA was 


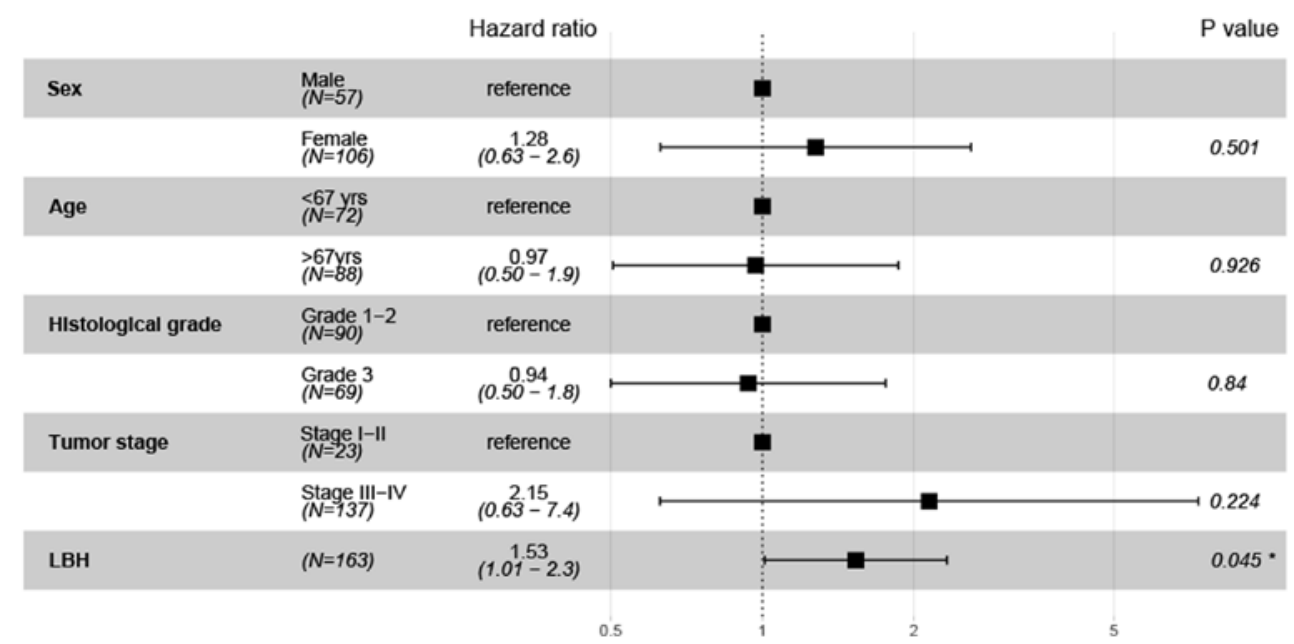

Figure 4. Multivariate Cox regression analyses for disease free survival. LBH, limb-bud and heart.

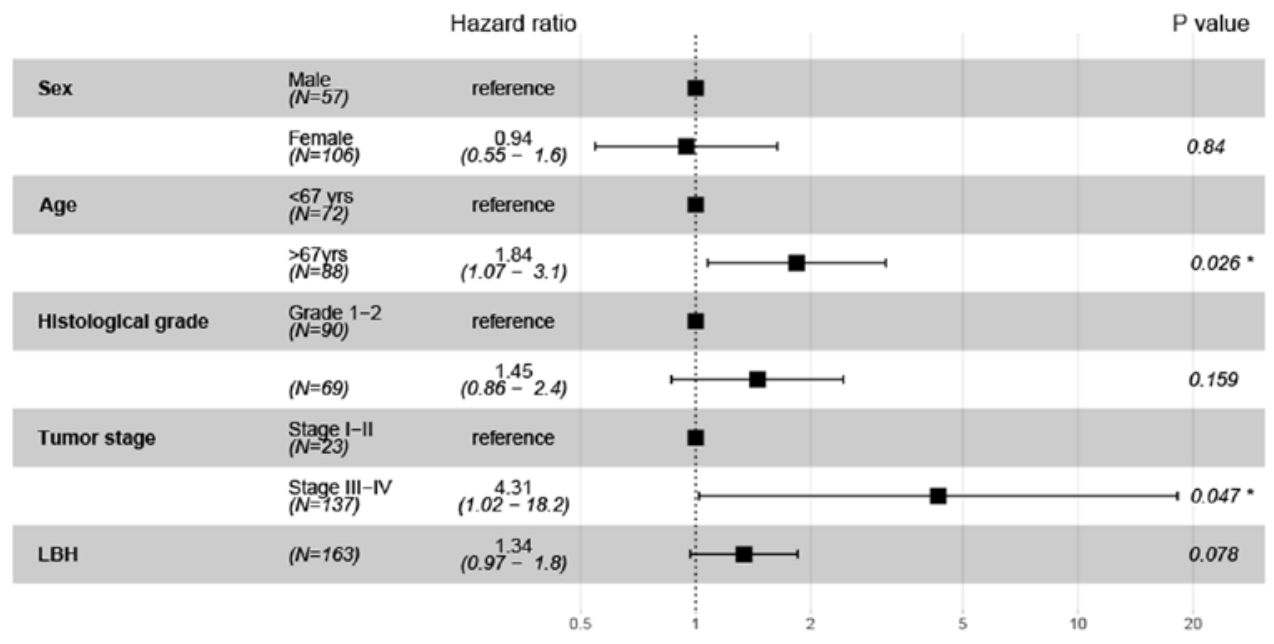

Figure 5. Multivariate Cox regression analyses for overall survival. LBH, limb-bud and heart.

assessed. Data of patients with GITA obtained from the TCGA database were first divided into the low- and high-expression groups. Kaplan-Meier survival analyses reported that patients in the high expression group exhibited significantly shorter OS and DFS compared with those in the low expression group. This result was consistent with data obtained using the Kaplan-Meier plotter database. Multivariate Cox analysis indicated that LBH was an independent prognostic factor of DFS, but not of OS. Age (>67 years) and stages T3-4 were found to be independent predictors of unfavorable prognosis in patients with GITA.

The results also revealed that the genes that were co-expressed with LBH in GITA were particularly enriched in the 'collagen-containing ECM', 'ECM structural constituent', 'ECM organization' and 'ECM-receptor interaction and focal adhesion'. ECM organization has been demonstrated to be associated with cell migration-related GO processes, which are linked with tumor metastasis (28), whilst ECM-receptor interaction and focal adhesion are pathways associated with metastasis (28). Additionally, LHB was reported to be involved in the PI3K-AKT signaling pathway, which has been demonstrated to be connected with the cell proliferation (29).
The PI3K-AKT pathway is the primary signaling pathway downstream of multiple growth factor receptors and is one of the most active signaling pathways in human tumors. Through the phosphorylation of the PI3K and AKT proteins, tumor cell proliferation and malignant transformation are promoted whereas tumor cell apoptosis is inhibited $(30,31)$. Consequently, PI3K-AKT inhibitors are widely used in cancer treatment (32). Several previous studies have reported the role of the PI3K-AKT pathway in promoting GC proliferation and invasion $(32,33)$. Therefore, the present study hypothesized that LBH may promote the malignant proliferation of GITA through the PI3K-AKT pathway. The results suggest that LBH serves an oncogenic function in GITA and cn be applied as a potential biomarker of disease prognosis.

However, the present study is not consistent with previous studies conducted in human nasopharyngeal cancer models and the prostate cancer cell line PC $3 \mathrm{M}$, which indicated that LBH is a tumor suppressor $(14,33)$. LBH expression was found to be downregulated in prostate cancer tissues and cell lines compared with that in healthy prostate epithelial cells (33). In addition, LBH overexpression was revealed to inhibit PC3M cell proliferation and tumor growth by inducing cell cycle 
A

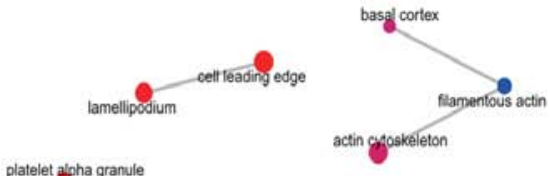

platelet algha granule
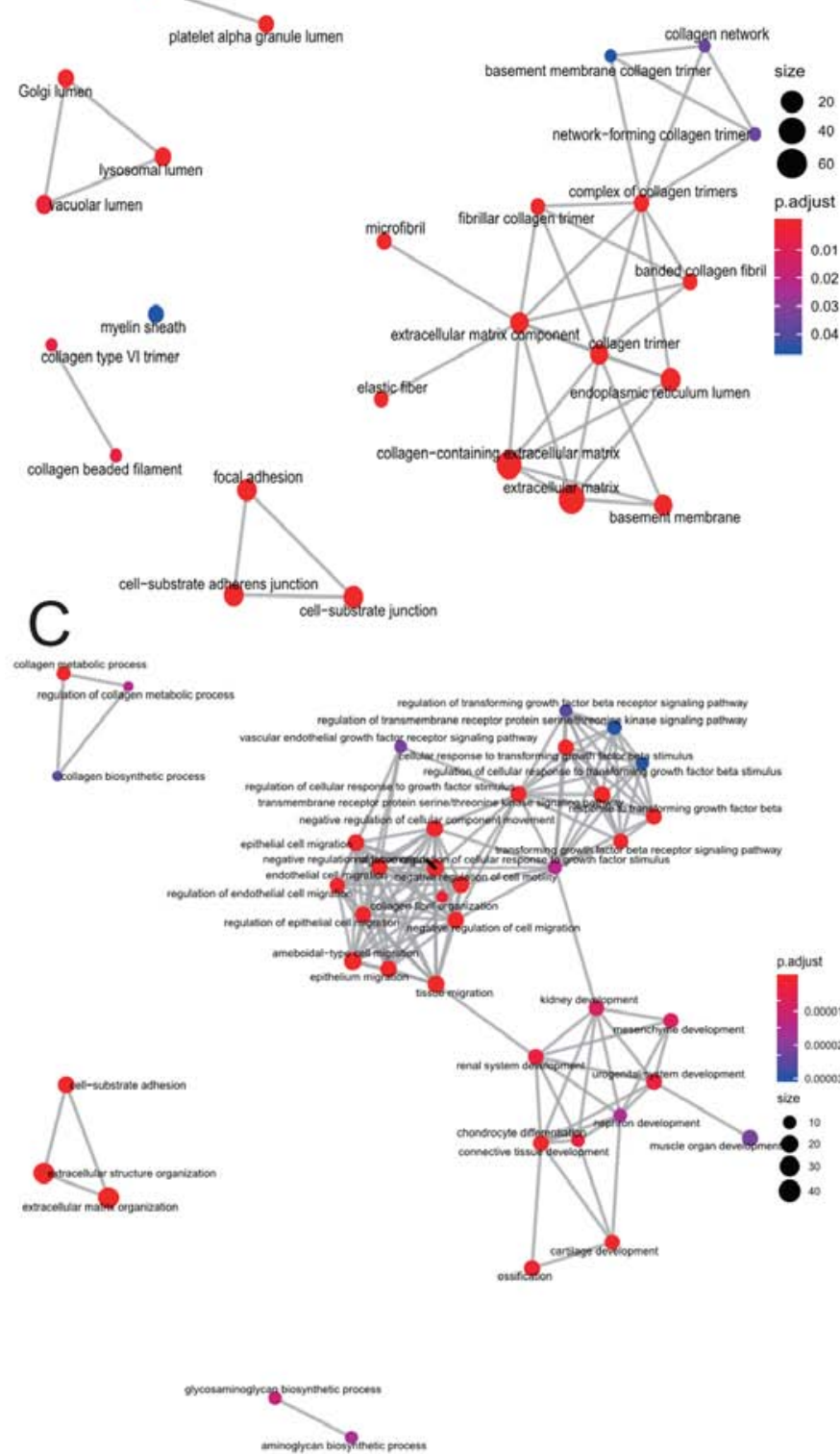

B

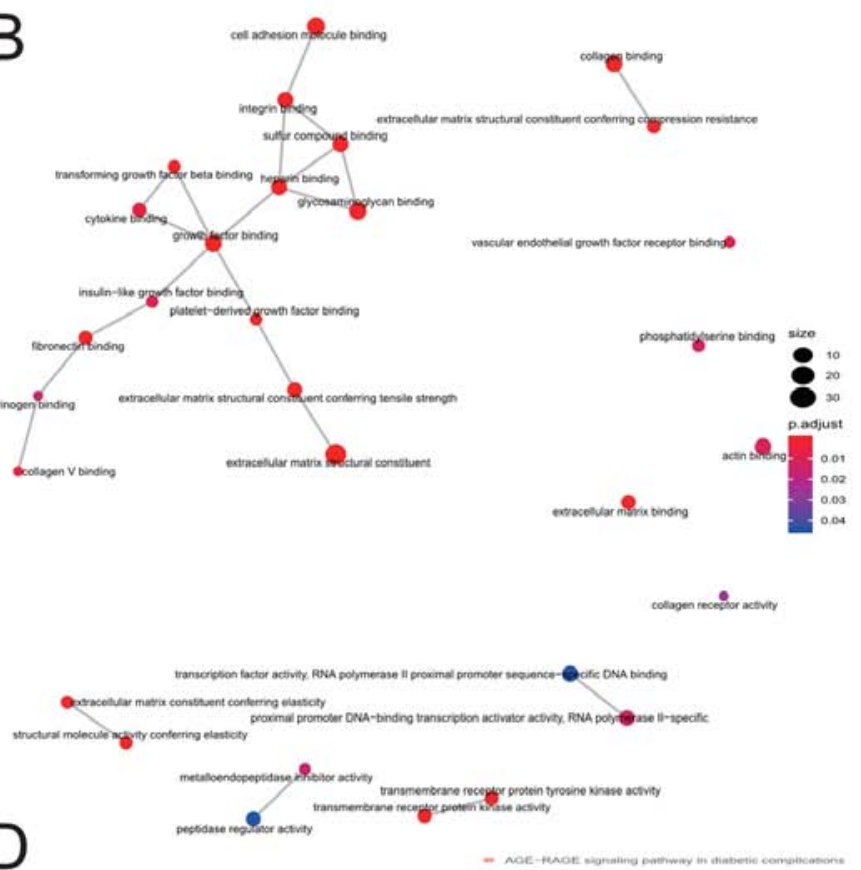

D

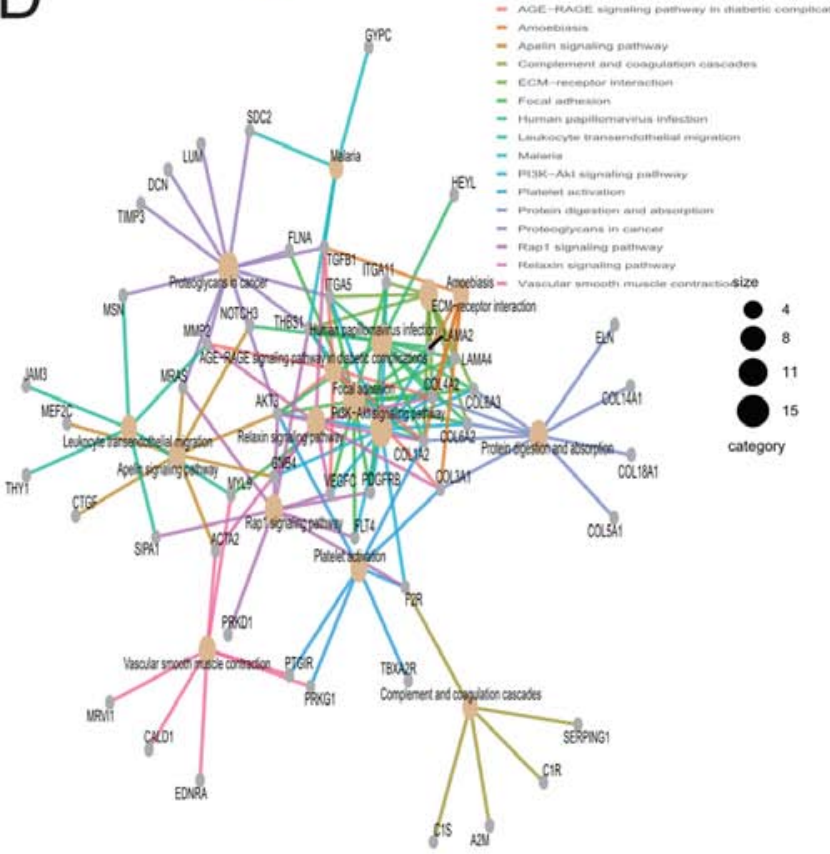

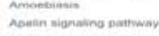

Figure 6. Functional Enrichment Analysis of the LBH co-expression network. The gene ontology terms in LBH and its co-expressed genes in the (A) cellular component, (B) molecular function and (C) biological process categories. (D) The network of LBH and its co-expressed genes in the Kyoto Encyclopedia of Genes and Genomes pathway. Panels A-C were generated using R software. LBH, limb-bud and heart; p.adjust, adjusted P-value.

arrest via downregulation of cyclin D1 and cyclin E2 gene expression (33). Therefore, it should be noted that LBH can function both as an oncogene and a tumor suppressor gene.

However, the current study has limitations. Firstly, the sample size analyzed was small. Secondly, the results of the enrichment analysis require further research to determine the potential molecular mechanisms by which $\mathrm{LBH}$ regulate GITA.

In summary, the present study demonstrated that LBH is highly expressed in GITA, where LBH overexpression predicted worse prognosis. LBH was an independent DFS predictor in GITA. Furthermore, the co-expressed genes enriched by LBH are associated with the migration, proliferation and metastasis of tumors. Therefore, LBH may be a potential prognostic biomarker and a therapeutic target for GITA.

\section{Acknowledgements}

Not applicable.

\section{Funding}

No funding was received. 


\section{Availability of data and materials}

The datasets generated and/or analyzed during the current study are available in the Oncomine (oncomine.org) and TCGA (portal.gdc.cancer.gov).

\section{Authors' contributions}

SW and YH conceived the study and performed bioinformatic analysis. JC collected the data and drafted the manuscript or revising it critically for important intellectual content. YY performed the experiments and analyzed data. HL and WC prepared the figures and/or tables and interpreted the data. All authors read and approved the final manuscript and agree to be accountable for all aspects of the work in ensuring that questions related to the accuracy or integrity of any part of the work are appropriately investigated and resolved.

\section{Ethics approval and consent to participate}

Not applicable.

\section{Patient consent for publication}

Not applicable.

\section{Competing interests}

The authors declare that they have no competing interests.

\section{References}

1. Siegel RL, Miller KD and Jemal A: Cancer Statistics, 2020. CA Cancer J Clin 0: 1-24, 2020.

2. Bray F, Ferlay J, Soerjomataram I, Siegel RL, Torre LA and Jemal A: Global cancer statistics 2018: GLOBOCAN estimates of incidence and mortality worldwide for 36 cancers in 185 countries. CA Cancer J Clin 68: 394-424, 2018.

3. Wadhwa R, Song S, Lee JS, Yao Y, Wei Q and Ajani JA: Gastric cancer-molecular and clinical dimensions. Nat Rev Clin Oncol 10: 643-655, 2013.

4. Ang TL and Fock KM: Clinical epidemiology of gastric cancer. Singapore Med J 55: 621-628, 2014.

5. Lauren P: The two histological main types of gastric carcinoma: Diffuse and so called intestinal type carcinoma. An attempt at a histo clinical classification. Acta Pathol Microbiol Scand 64: 31-49, 1965.

6. Figueiredo C, Costa S, Karameris A and Machado JC: Pathogenesis of Gastric Cancer. Helicobacter 20 (Suppl 1): 30-35, 2015.

7. Park YH and Kim N: Review of atrophic gastritis and intestinal metaplasia as a premalignant lesion of gastric cancer. J Cancer Prev 20: 25-40, 2015.

8. Briegel KJ: Embryonic transcription factors in human breast cancer. IUBMB Life 58: 123-132, 2006.

9. Ben-Porath I, Thomson MW, Carey VJ, Ge R, Bell GW, Regev A and Weinberg RA: An embryonic stem cell-like gene expression signature in poorly differentiated aggressive human tumors. Nat Genet 40: 499-507, 2008.

10. Francis R, Guo H, Streutker C, Ahmed M, Yung T, Dirks PB, He HH and Kim TH: Gastrointestinal transcription factors drive lineage specific developmental programs in organ specification and cancer. Sci Adv 5: eaax8898, 2019.

11. Raghoebir L, Bakker ER, Mills JC, Swagemakers S, Kempen MB, Munck AB, Driegen S, Meijer D, Grosveld F, Tibboel D, et al: SOX2 redirects the developmental fate of the intestinal epithelium toward a premature gastric phenotype. J Mol Cell Biol 4: 377-385, 2012.

12. Briegel KJ and Joyner AL: Identification and characterization of Lbh, a novel conserved nuclear protein expressed during early limb and heart development. Dev Biol 233: 291-304, 2001.
13. Rieger ME, Sims AH, Coats ER, Clarke RB and Briegel KJ: The embryonic transcription cofactor LBH is a direct target of the Wnt signaling pathway in epithelial development and in aggressive basal subtype breast cancers. Mol Cell Biol 30: 4267-4279, 2010.

14. Liu Q, Guan X, Lv J, Li X, Wang Y and Li L: Limb-bud and Heart (LBH) functions as a tumor suppressor of nasopharyngeal carcinoma by inducing G1/S cell cycle arrest. Sci Rep 5: 7626, 2015.

15. Deng M, Yu R, Wang S, Zhang Y, Li Z, Song H, Liu B, Xu L, Wang $X$, Zhang Z, et al: Limb-Bud and heart attenuates growth and invasion of human lung adenocarcinoma cells and predicts survival outcome. Cell Physiol Biochem 47: 223-234, 2018.

16. Kruppa J and Jung K: Automated multigroup outlier identification in molecular high-throughput data using bagplots and gemplots. BMC Bioinformatics 18: 232, 2017.

17. Chen J, Hu B, Wang W, Qian XJ, Shan BJ and He YF: A six-microRNA signature to predict outcomes of patients with gastric cancer. FEBS Open Bio 9: 538-547, 2019.

18. Nagy Á, Lánczky A, Menyhárt O and Győrffy B: Validation of miRNA prognostic power in hepatocellular carcinoma using expression data of independent datasets. Sci Rep 8: 9227, 2018.

19. Jing JJ, Wang ZY, Li H, Sun LP and Yuan Y: Key elements involved in Epstein-Barr virus-associated gastric cancer and their network regulation. Cancer Cell Int 18: 146, 2018.

20. Chen X, Leung SY, Yuen ST, Chu KM, Ji J, Li R, Chan AS, Law S, Troyanskaya OG, Wong J, et al: Variation in gene expression patterns in human gastric cancers. Mol Biol Cell 14: 3208-3215, 2003.

21. D'Errico M, de Rinaldis E, Blasi MF, Viti V, Falchetti M, Calcagnile A, Sera F, Saieva C, Ottini L, Palli D, et al: Genome-wide expression profile of sporadic gastric cancers with microsatellite instability. Eur J Cancer 45: 461-469, 2009.

22. Cho JY, Lim JY, Cheong JH, Park YY, Yoon SL, Kim SM, Kim SB, Kim H, Hong SW, Park YN, et al: Gene expression signature-based prognostic risk score in gastric cancer. Clin Cancer Res 17: 1850-1857, 2011.

23. Kahn M: Can we safely target the WNT pathway? Nat Rev Drug Discov 13: 513-532, 2014.

24. Clevers $\mathrm{H}$ and Nusse R: Wnt/ $\beta$-catenin signaling and disease. Cell 149: 1192-1205, 2012.

25. Ashad-Bishop K,Garikapati K, Lindley LE, Jorda M and Briegel KJ: Loss of Limb-Bud-and-Heart (LBH) attenuates mammary hyperplasia and tumor development in MMTV-Wnt1 transgenic mice. Biochem Biophys Res Commun 508: 536-542, 2019.

26. Lindley LE, Curtis KM, Sanchez-Mejias A, Rieger ME, Robbins DJ and Briegel KJ: The WNT-controlled transcriptional regulator LBH is required for mammary stem cell expansion and maintenance of the basal lineage. Development 142: 893-904, 2015.

27. Chen J, Huang C, Chen K, Li S, Zhang X, Cheng J, Cai M and Xiao Y: Overexpression of $\mathrm{LBH}$ is associated with poor prognosis in human hepatocellular carcinoma. OncoTargets Ther 11: 441-448, 2018.

28. Liu H, Wu N, Zhang Z, Zhong X, Zhang H, Guo H, Nie Y and Liu Y: Long Non-coding RNA LINC00941 as a potential biomarker promotes the proliferation and metastasis of gastric cancer. Front Genet 10: 5, 2019.

29. Lu Y, Li L, Wu G, Zhuo H, Liu G and Cai J: Effect of PI3K/Akt signaling pathway on PRAS40Thr246 phosphorylation in gastric cancer cells. Iran J Public Health 48: 2196-2204, 2019.

30. Hu M, Zhu S, Xiong S, Xue X and Zhou X: MicroRNAs and the PTEN/PI3K/Akt pathway in gastric cancer (Review). Oncol Rep 41: 1439-1454, 2019.

31. Jia L, Zhu Z, Li H and Li Y: Shikonin inhibits proliferation, migration, invasion and promotes apoptosis in NCI-N87 cells via inhibition of PI3K/AKT signal pathway. Artif Cells Nanomed Biotechnol 47: 2662-2669, 2019.

32. Zhang C, Lin X, Zhao Q, Wang Y, Jiang F, Ji C, Li Y, Gao J, Li J and Shen L: YARS as an oncogenic protein that promotes gastric cancer progression through activating PI3K-Akt signaling. J Cancer Res Clin Oncol 146: 329-342, 2020.

33. Liu Q, Li E, Huang L, Cheng M and Li L: Limb-bud and heart overexpression inhibits the proliferation and migration of $\mathrm{PC} 3 \mathrm{M}$ Cells. J Cancer 9: 424-432, 2018.

This work is licensed under a Creative Commons Attribution-NonCommercial-NoDerivatives 4.0 International (CC BY-NC-ND 4.0) License. 